\title{
Estado nutricional de niños con estenosis esofágica secundaria a quemadura por cáusticos, alimentados por gastrostomía en el Hospital Civil de Guadalajara
}

\author{
Nutritional status of children with esophageal stenosis secondary to burns by caustics, \\ with gastrostomy management in the Civil Hospital of Guadalajara
}

\author{
Martha del R. Cornejo-Villa*, Andrea Torres-Rojas, María A. Soto-Blanquel y Juan L. Soto-Mancilla \\ Departamento Institucional de Nutrición Clínica y Cirugía Pediátrica, Organismo Público Descentralizado (OPD), Hospital Civil de Guadalajara Fray \\ Antonio Alcalde, Guadalajara, Jalisco, México
}

\section{Resumen}

Antecedentes: La ingestión de cáusticos constituye un problema grave en la edad pediátrica que puede ocasionar lesiones en el esófago que imposibiliten la alimentación vía oral. La nutrición por gastrostomía resulta una vía segura para garantizar una evolución adecuada del estado nutricional, así como favorecer el crecimiento y el desarrollo del paciente. Objetivo: Evaluar el estado nutricional de niños con estenosis esofágica secundaria a ingestión de cáusticos, posterior a 6 meses de la colocación de gastrostomía como vía de alimentación. Método: Estudio descriptivo, longitudinal, ambispectivo, con una muestra de 20 pacientes. El estado nutricional previo y posterior a la colocación de gastrostomía se obtuvo mediante mediciones antropométricas e índices pediátricos. Análisis estadístico: $t$ de Student. Resultados: Los índices antropométricos de peso para la edad (P/E) e índice de masa corporal para la edad (IMC/E) reflejan una mejoría estadísticamente significativa en el estado nutricional a lo largo de 6 meses de tratamiento con gastrostomía, no siendo así para la talla para la edad (T/E). Conclusiones: El estado nutricional evoluciona adecuadamente a partir del manejo con gastrostomía en estos pacientes.

Palabras clave: Cáustico. Estado nutricional. Estenosis esofágica. Gastrostomía.

\begin{abstract}
Background: The ingestion of caustic substances constitutes a serious problem in the pediatric age that can cause injuries in the esophagus that may require oral feeding. Gastrostomy nutrition is a safe way to guarantee an adequate evolution of the nutritional status, as well patient's growth and development. Objective: To evaluate the nutritional status of children with esophageal stenosis, secondary to caustic ingestion, after 6 months of gastrostomy placement as a feeding route. Method: A descriptive, longitudinal ambispective study was carried out, with a sample of 20 patients. Nutritional status before and after gastrostomy placement; was obtained through anthropometric measurements and pediatric indexes. Statistical analysis: $t$-test. Results: The anthropometric indexes of W/A and BMI/A reflect a statistically significant improvement in nutritional status over 6 months of gastrostomy treatment, but not for H/A. Conclusion: The nutritional status evolves adequately from gastrostomy management in these patients.
\end{abstract}

Key words: Caustic. Nutritional status. Esophageal stenosis. Gastrostomy.

\footnotetext{
Correspondencia:

*Martha del R. Cornejo-Villa

Ejército Constitucionalista, 4017

Fecha de recepción: 14-10-2019

Cir Cir. 2020;88(6):726-731

C.P. 45120, Zapopan, Jalisco, México

Fecha de aceptación: 02-04-2020

Contents available at PubMed

E-mail: rocio.cornejov@ hotmail.com

DOI: $10.24875 / C I R U .20001653$

www.cirugiaycirujanos.com

0009-7411/@ 2020 Academia Mexicana de Cirugía. Publicado por Permanyer. Este es un artículo open access bajo la licencia CC BY-NC-ND (http://creativecommons.org/licenses/by-nc-nd/4.0/).
} 


\section{Introducción}

El esófago es un órgano tubular y longitudinal que se extiende desde la faringe hasta el estómago, cuyo objetivo es transportar el bolo alimenticio. Comienza y termina en dos estructuras, el esfínter esofágico superior y el esfínter esofágico inferior, que lo independizan de la faringe y del estómago'.

La longitud media en el adulto es de $22-25 \mathrm{~cm}$ : 5-6 $\mathrm{cm}$ para el esófago cervical, $16-18 \mathrm{~cm}$ para la porción torácica y $3 \mathrm{~cm}$ para el segmento abdominal. La Iongitud del esófago al nacer es de unos 8-10 cm y mide unos $19 \mathrm{~cm}$ a los 15 años ${ }^{2}$.

Por cáustico se conoce a toda sustancia que produce la destrucción de los tejidos expuestos al atacar a las membranas celulares, causando necrosis. Los ácidos cáusticos, con un $\mathrm{pH}<2$, como el ácido muriático y el ácido clorhídrico, van a producir necrosis coagulativa, mientras que los álcalis cáusticos, que tienen un $\mathrm{pH}$ entre 11.5 y 14 , como el hidróxido de sodio (sosa cáustica) y el amoníaco, producen necrosis licuefactiva. Si estas sustancias se ingieren, se pueden producir lesiones graves en el aparato gastrointestinal ${ }^{3}$. Las quemaduras por álcalis son más frecuentes que por ácidos debido a que su uso es más habitual en el hogar ${ }^{4}$. En este estudio, los pacientes ingresaron al hospital por ingestión de hidróxido de sodio.

Los ácidos producen necrosis por coagulación proteica, existe pérdida de agua y se forma una escara firme y protectora que dificulta la penetración, salvo en concentraciones elevadas 5 . Los álcalis inducen necrosis por licuefacción, con desnaturalización de las proteínas, saponificación de las grasas que permite la penetración profunda a la submucosa y la muscular, resultando en trombosis capilar y fibrosis del tejido o incluso perforación. La retención de agua aumenta la quemadura por hidratación y esto favorece la profundidad de las lesiones. Los álcalis principalmente lesionan el tracto gastrointestinal proximal, incluyendo la boca, la faringe, el esófago y el estómago ${ }^{6}$.

Ya que la ingestión de ácidos causa un intenso dolor orofaríngeo, generalmente se consumen en pequeños volúmenes comparados con las sustancias alcalinas. Los ácidos pasan rápido por el esófago, resultando en una menor frecuencia y un menor grado de lesiones esofágicas ${ }^{7}$.

Las quemaduras esofágicas son lesiones producidas por la ingestión de sustancias cáusticas y se encuentran dentro de los accidentes más recurrentes en la edad pediátrica ${ }^{4}$. En México, alrededor de un $5 \%$ de todos los accidentes domésticos se producen por la ingestión de sustancias cáusticas, ya que existe la facilidad para adquirir soluciones de álcalis y ácidos para su uso en el hogar, lo que ha producido un aumento de la frecuencia de quemaduras y de la disminución del consumo de alimentos ${ }^{8}$.

El $50 \%$ de los casos con quemadura esofágica grave (grado IIB y IIIA) ${ }^{9}$ tienen pronóstico de presentar una estenosis esofágica mayor del $90 \%$, que impida la alimentación oral por períodos largos de tiempo ${ }^{10}$. En el Hospital Civil de Guadalajara Fray Antonio Alcalde se atienden en promedio 22 casos al año.

El tratamiento consiste en dilataciones del esófago para recuperar el calibre y que se permita la alimentación. Sin embargo, en ocasiones, cuando el grado de lesión es mayor y existen perforaciones esofagogástricas o disfagia muy intensa, se considera realizar una gastrostomía; de igual manera cuando se impida una alimentación adecuada, con menos del $70 \%$ de los requerimientos energéticos por vía oral ${ }^{11,12}$. El tratamiento nutricional es indispensable, ya que de esta manera se puede asegurar el aporte del requerimiento energético necesario de la manera más fisiológica y con ello evitar la desnutrición calórico-proteica ${ }^{13}$.

A pesar de que es un problema recurrente en la edad pediátrica, existe poca información respecto a esta patología y su impacto en el estado nutricional, así como sobre el pronóstico del paciente. La gran mayoría de los datos disponibles no abordan dicho aspecto, aunado a que no hay un factor de estrés establecido para este tipo de quemadura, por lo que no es posible calcular de manera exacta el requerimiento calórico de estos pacientes.

El objetivo de este estudio fue identificar la evolución del estado nutricional de pacientes pediátricos con estenosis esofágica secundaria a quemadura por cáusticos alimentados por gastrostomía en el Hospital Civil de Guadalajara Fray Antonio Alcalde de 2014 a 2017.

\section{Método}

Estudio descriptivo, longitudinal y ambispectivo. La población a la cual se tuvo acceso fue de 20 pacientes pediátricos con diagnóstico de estenosis esofágica secundaria a quemadura por cáusticos, alimentados por gastrostomía (técnica quirúrgica), que fueron atendidos en el Hospital Civil de Guadalajara Fray Antonio Alcalde de 2014 a 2017. Para la obtención del tamaño muestral se consideró una muestra no 
probabilística por juicio de experto tipo censal. La recolección de los datos fue de mayo de 2017 a octubre de 2018.

El objetivo general del estudio fue evaluar el estado nutricional posterior a 6 meses de la colocación de la gastrostomía como vía de alimentación. Los objetivos específicos fueron conocer la incidencia de diagnóstico de quemadura esofágica secundaria a ingestión de cáusticos en pacientes pediátricos, identificar la cantidad de pacientes que recibieron tratamiento nutricional y conocer la incidencia del uso de gastrostomía en esta patología.

La presente investigación fue aprobada y revisada por el Comité de Ética del Hospital Civil de Guadalajara Fray Antonio Alcalde, bajo el número de registro 277/17. Se siguieron los principios de la Declaración de Helsinki y La Ley General de Salud. Esta investigación se consideró sin riesgo debido a que solo requirió la extracción de información de las bases de datos del servicio de nutrición clínica pediátrica, así como de las endoscopias realizadas por el servicio de cirugía pediátrica de los años 2014 a 2017, y no fue necesario utilizar un consentimiento informado para la realización de la investigación. Sin embargo, los padres o tutores previamente otorgaron el consentimiento a los servicios para las intervenciones pertinentes.

Se recolectó información acerca de los pacientes con estenosis esofágica secundaria a ingesta de cáusticos con un tratamiento nutricional durante 6 meses por gastrostomía. No se incluyeron aquellos alimentados por vía oral y se eliminaron los que no contaban con la información indispensable para el estudio.

Se obtuvieron datos de las endoscopias realizadas desde enero de 2014 hasta diciembre de 2017 del servicio de cirugía pediátrica del Hospital Civil de Guadalajara Fray Antonio Alcalde, así como la información de la evaluación del estado nutricional del ingreso hospitalario y de tres consultas subsecuentes (1, 3 y 6 meses posteriores a la quemadura esofágica), y del abordaje nutricional por parte del servicio de nutrición clínica pediátrica.

Se registraron los siguientes datos: nombre completo del paciente, registro hospitalario, fecha de ingreso hospitalario, fecha de nacimiento, diagnóstico al ingreso, fecha de la quemadura, fecha de colocación y retiro de la gastrostomía, fecha de las primeras tres endoscopias con su respectivo diagnóstico endoscópico, y plan médico a seguir. De igual forma, fueron necesarios la edad en meses, el peso, la talla, el índice de masa corporal (IMC) y las calorías del tratamiento del ingreso hospitalario y las primeras tres consultas para evaluar el progreso de los pacientes.

La intervención nutricional del paciente comenzó en su ingreso hospitalario con nutrición parenteral y ayuno por vía oral, hasta el momento de la colocación de la gastrostomía, y posteriormente se hizo la transición a nutrición enteral hasta alcanzar el requerimiento energético total. El tratamiento nutricional proporcionado por parte del servicio de nutrición pediátrica fue un $20 \%$ mayor que el aporte calórico habitual del paciente. El aporte energético proporcionado a los pacientes depende de su estado nutricional, otorgando aproximadamente $100-200 \mathrm{kcal} / \mathrm{kg} / \mathrm{día}^{14}$, con un aporte proteico de 2.0-3.0 g proteína/kg/día.

Se utilizaron los programas WHO Anthro versión 3.2.2 y WHO Anthro Plus versión 1.0.4 para la obtención de la puntuación $Z$ del peso para la edad (P/E), la talla para la edad (T/E) y el IMC para la edad (IMC/E). Se utilizó estadística descriptiva incluyendo media, desviación estándar, valor mínimo, valor máximo y proporciones por medio de Microsoft Office Excel 2013. Se realizó el análisis estadístico inferencial con el paquete estadístico IBM SPSS versión 23. El método estadístico utilizado fue la prueba t de Student, considerando significativas las diferencias cuando $p<0.05$.

\section{Resultados}

Durante el periodo de enero de 2014 a agosto de 2017 se registraron 92 pacientes que ingresaron con diagnóstico médico de quemadura esofágica secundaria a ingesta de cáusticos en el servicio de cirugía pediátrica del Hospital Civil de Guadalajara Fray Antonio Alcalde, de los cuales solo 30 (32.6\%) recibieron tratamiento nutricional por parte del servicio de nutrición pediátrica, y de ellos 26 (28.2\%) requirieron colocación de gastrostomía y seis fueron eliminados ya que no cumplían con la información indispensable para la investigación.

El grupo de estudio estuvo conformado por 20 pacientes con estenosis esofágica grave y quemaduras esofágicas de grado mayor de IIB y IIIA según los criterios endoscópicos de Zargar, lo que les impidió cubrir más del $70 \%$ del requerimiento energético total por vía oral; dos de ellos presentaban quemadura gástrica IIA en la primera endoscopia. En cuanto al sexo, el $75 \%$ eran varones y el $25 \%$ eran mujeres. La edad promedio fue de 46 meses (3.8 años), con un intervalo de 20.8 meses (1.7 años) a 97.3 meses (8.1 años). 


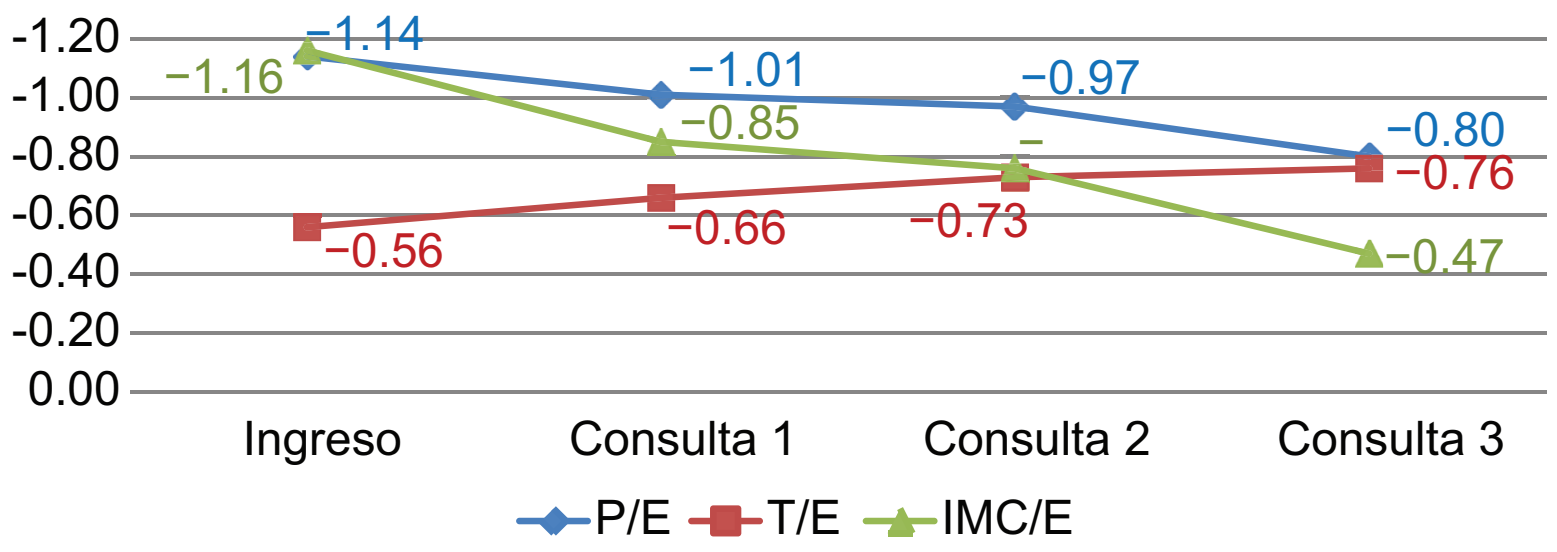

Figura 1. Puntuación Z de los índices antropométricos en las consultas nutricionales. IMC/E: índice de masa corporal para la edad; P/E: peso para la edad; T/E: talla para la edad.

Tabla 1. Clasificación de la puntuación $Z$ de los índices antropométricos al ingreso hospitalario y en la consulta 3

\begin{tabular}{|c|c|c|c|c|c|c|}
\hline \multirow[t]{2}{*}{ DE } & \multicolumn{2}{|c|}{$\mathrm{P} / \mathrm{E}$} & \multicolumn{2}{|c|}{$T / E$} & \multicolumn{2}{|c|}{ IMC/E } \\
\hline & Ingreso(\%) & Consulta 3 (\%) & Ingreso (\%) & Consulta $3(\%)$ & Ingreso (\%) & Consulta $3(\%)$ \\
\hline +3.0 y mayor & 0 & 0 & 0 & 0 & 0 & $65(n=13)$ \\
\hline$+2.0 a+2.99$ & 0 & 0 & $5(n=1)$ & 0 & $5(n=1)$ & $30(n=6)$ \\
\hline$+1.01 \mathrm{a}+1.99$ & $5(n=1)$ & 0 & $5(n=1)$ & $5(n=1)$ & $5(n=1)$ & 0 \\
\hline$+1.0 \mathrm{a}-1.0$ & $25(n=5)$ & $60(n=12)$ & $55(n=11)$ & $60(n=12)$ & $40(n=8)$ & 0 \\
\hline$-1.01 \mathrm{a}-1.99$ & $50(n=10)$ & $25(n=5)$ & $20(n=4)$ & $15(n=3)$ & $20(n=4)$ & $5(n=1)$ \\
\hline$-2.0 a-2.99$ & $10(n=2)$ & $10(n=2)$ & $10(n=2)$ & $15(n=3)$ & $30(n=6)$ & 0 \\
\hline -3.0 y menor & $10(n=2)$ & $5(n=1)$ & $5(n=1)$ & $5(n=1)$ & 0 & 0 \\
\hline
\end{tabular}

DE: desviación estándar; IMC/E: índice de masa corporal para la edad; P/E: peso para la edad; T/E: talla para la edad.

En la figura 1 se muestra la evolución de la puntuación $Z$ de los índices antropométricos con los que se evaluó el estado nutricional (P/E, T/E e IMC/E) en las consultas. En la tabla 1 se encuentran los resultados de los índices del estado nutricional entre el ingreso hospitalario y la última consulta.

La puntuación Z del P/E inicial del 25\% se ubicó dentro de la normalidad y del $50 \%$ en bajo peso. Se obtuvo una $p=0.037$ al comparar las medias. En comparación con el P/E final, el $60 \%$ indicó normalidad y el $25 \%$ bajo peso. La puntuación $Z$ de la T/E inicial del $60 \%$ tuvo un diagnóstico de normalidad y en el indicador final fue un $65 \%$. La puntuación $Z$ del IMC/E inicial en el $40 \%$ indicó normalidad, en el 50\% se encontró emaciación y en el 10\% sobrepeso. La puntuación $Z$ del IMC/E final en el $65 \%$ se ubicó dentro de la normalidad, el 30\% presentaron emaciación y el $5 \%$ sobrepeso. Se obtuvo una $p=0.021$ al comparar las medias.

En cuanto al abordaje nutricional con gastrostomía, se observó que el promedio de duración fue de 14.4 \pm 10.5 meses. El tiempo transcurrido entre la colocación de la gastrostomía y el momento de la quemadura depende del grado de estenosis esofágica, de la clínica, de la evolución y del seguimiento de cada paciente. En este estudio se observa que se recurrió a la colocación de esta en los primeros 10 días en aquellos que presentaron un porcentaje de estenosis mayor del $80 \%$.

\section{Discusión}

En México, los accidentes forman parte de las principales causas de mortalidad en los niños menores 
de 5 años, lo que refleja una pobre cultura en su prevención $n^{15}$. Actualmente no existe información relacionada con la epidemiología de la ingestión por cáusticos en nuestro país, a pesar de que el número de casos reportados es relativamente alto, lo cual se puede constatar en diferentes estudios.

En un estudio acerca de factores sociodemográficos asociados a la ingesta de cáusticos en niños y adolescentes durante el año 2006 realizado en un hospital pediátrico en Guadalajara, México, se estudiaron 94 pacientes con una edad promedio de 3.2 años y 59 de ellos (62.8\%) fueron niños. Se encontró que las variables sociodemográficas significativamente asociadas fueron la edad menor de 6 años, el sexo masculino, la madre menor de 30 años, el bajo nivel educativo de la madre, la madre que trabaja fuera de casa y niños con padres divorciados ${ }^{16}$. De igual manera, la ENSANUT 2012 describe que en el ámbito nacional existe una prevalencia de accidentes más alta en los niños que en las niñas (5.3 y $3.4 \%$, respectivamente $)^{17}$. En el presente estudio se observó una tendencia similar en cuanto al riesgo que presentan los varones de ingerir accidentalmente una sustancia cáustica.

La gravedad del daño esofágico secundario a la quemadura por agentes cáusticos se refleja en la incidencia de estenosis esofágicas graves que conllevan la imposibilidad de alimentación por vía oral, por lo cual es necesaria la colocación de gastrostomía en un $28.2 \%$ de los pacientes.

La puntuación Z del IMC/E del $30 \%$ de los pacientes se ubicó debajo de -2 desviaciones estándar (DE), el $20 \%$ debajo de $-1 \mathrm{DE}$, el $10 \%$ se encontró con sobrepeso y el $30 \%$ con talla baja ( $10 \%$ con $-2 \mathrm{DE}$ ), lo cual contrasta con el estudio de Sánchez-Ramírez, et al. ${ }^{18}$ realizado en 2016 con 62 niños tratados en la Clínica de Esófago en la Unidad Médica de Alta Especialidad (UMAE), Hospital de Pediatría, México, al contar con un mayor porcentaje de niños con emaciación, menor con sobrepeso y un $40 \%$ de pacientes con un estado nutricional normal. Asimismo, más de la mitad de los pacientes se ubicaron por debajo del promedio en relación a los indicadores de brazo, lo que refleja una pérdida de las reservas grasas y musculares. Esto se puede considerar como un déficit en el estado nutricional de manera crónica. En comparación con las cifras reportadas por la ENSANUT 2012, existe una disparidad con respecto a los porcentajes nacionales de emaciación $(1.6 \%)$ y talla baja $(13.6 \%)^{17}$. Esto lleva a pensar que la población estudiada cuenta con una menor seguridad alimentaria previa al accidente, ya que la talla baja es reflejo de los efectos negativos acumulados a lo largo del tiempo.

Con respecto a la evolución del estado nutricional a partir del tratamiento por vía gastrostomía, en un estudio realizado en la UMAE Hospital de Pediatría, Ciudad de México, se estudiaron 97 pacientes con lesión esofágica grave por cáusticos ocurrida cerca de los 3 años. Se encontró que menos del $50 \%$ restablecieron y mantuvieron el estado nutricional con el empleo de gastrostomía o alimentación por vía oral con la administración de fórmulas poliméricas o artesanales $^{18}$. Sin embargo, en el presente estudio se observaron diferencias significativas en $P / E(p=0.037)$ e IMC/E ( $p=0.021)$. Al ingreso hospitalario, el $25 \%$ de los pacientes se ubicó dentro de la normalidad, y al finalizar el estudio el $60 \%$ se encontró en normopeso, a pesar de que se trata de una forma de alimentación no convencional, pero con la que se puede cubrir de manera adecuada el requerimiento nutricional del niño. Comparando ambos estudios, aunque las quemaduras son del mismo tipo, las variables estudiadas fueron distintas. Sin embargo, en esta investigación se especifican la duración del tratamiento y la energía aportada, por lo que la diferencia del restablecimiento del estado nutricional puede deberse a la continuidad de consultas por 6 meses, al énfasis en la educación nutricional ofrecida a la madre y a brindar una fórmula polimérica casera con el $20 \%$ más de calorías que la ingesta habitual.

En un estudio realizado en diferentes hospitales pediátricos de Jalisco, en 2006, se estudiaron 94 pacientes con estenosis por cáusticos, de los cuales 18 recibieron nutrición enteral y el $50 \%$ tuvo resultados favorables con la gastrostomía ${ }^{16}$; también en esta investigación hubo mejoría en un $25 \%$ sobre el IMC y en un $35 \%$ en el peso corporal, lo cual corrobora que el aporte nutricional por gastrostomía es importante en este tipo de pacientes.

La ingestión de cáusticos en la edad pediátrica es alarmante, debido a que la recurrencia de este accidente habla de la falta de información dirigida específicamente a los padres y cuidadores, lo cual podría evitarse haciendo conciencia acerca de la importancia del correcto almacenamiento de sustancias corrosivas.

En cuanto al abordaje nutricional, el cálculo de los requerimientos resulta complejo debido a que no existe un factor de estrés específico para quemadura esofágica, pues es difícil determinar un valor único por la gravedad de la patología y el tipo de tratamiento. Sin embargo, al aumentar la ingesta habitual del 
paciente se logra alcanzar una mejora a largo plazo en el estado nutricional.

Es importante recalcar que, en el presente estudio, el tratamiento otorgado fue con fórmulas poliméricas caseras y no se utilizó ningún tipo de fórmula comercial para lograr una evolución en el estado nutricional.

Actualmente aún existen pocas referencias bibliográficas que hablen acerca del abordaje nutricional o del pronóstico de los niños con estenosis esofágica posterior a la ingestión de cáusticos, lo que invita a continuar las investigaciones para conocer más acerca de la situación en el país.

\section{Conclusión}

Existe poca información acerca de la estenosis esofágica secundaria a la ingestión de cáusticos, y sobre el tratamiento nutricional con gastrostomía y su evolución.

La ingestión de cáusticos constituye un problema grave y relativamente frecuente, el cual afecta en especial al sexo masculino de entre 2 y 8 años. En el periodo de 2014 a 2017 se encontraron 92 pacientes con diagnóstico de quemadura esofágica secundaria a cáusticos, de los cuales 30 (32.6\%) recibieron un abordaje por parte del servicio de nutrición pediátrica; $26(28.2 \%)$ requirieron el uso de gastrostomía por un tiempo promedio de 14.4 meses.

Los índices antropométricos P/E e IMC/E reflejan una mejoría estadísticamente significativa a lo largo de 6 meses de tratamiento con gastrostomía, no siendo así para T/E.

Por todo lo anterior, se concluye que el estado nutricional evoluciona adecuadamente con el manejo con gastrostomía en estos pacientes.

\section{Financiamiento}

Los autores declaran que no hubo fuentes de financiamiento.

\section{Conflicto de intereses}

Los autores declaran no tener conflicto de intereses relacionados con el presente manuscrito.

\section{Responsabilidades éticas}

Protección de personas y animales. Los autores declaran que para esta investigación no se han realizado experimentos en seres humanos ni en animales.

Confidencialidad de los datos. Los autores declaran que han seguido los protocolos de su centro de trabajo sobre la publicación de datos de pacientes.

Derecho a la privacidad y consentimiento informado. Los autores han obtenido el consentimiento informado de los pacientes y/o sujetos referidos en el artículo. Este documento obra en poder del autor de correspondencia.

\section{Bibliografía}

1. Sociedad Española de Otorrinolaringología y Patología Cérvico-Facial. Otorrinolaringología. Madrid: Médica Panamericana; 2011.

2. Viswanatha B. Esophagus anatomy. Medscape. 2015 Jul. Disponible en: https://emedicine.medscape.com/article/1948973-overview.

3. Mencías E. Intoxicación por cáusticos. An Sist Sanit Navarra. 2003;26:191-207.

4. Millar AJ, Cox SG. Caustic injury of the oesophagus. Pediatr Surg Int. 2011;31:111-21.

5. Asociación Española de Pediatría. Protocolos de gastroenterología, hepatología y nutrición. 2. a ed. Madrid: Ergón; 2011.

6. Kurowski JA, Kay, M. Caustic ingestions and foreign bodies ingestions in pediatric patients. Pediatr Clin North Am. 2017;64:507-24.

7. Contini S, Scarpignato C. Caustic injury of the upper gastrointestinal tract: a comprehensive review. World J Gastoenterol. 2013;19:3918-30.

8. Asociación Española de Gastroenterología. Esofagitis por cáusticos. En: Montoro MA, García JC, editores. Gastroenterología y hepatología: problemas comunes en la práctica clínica. 2. ${ }^{\text {a }}$ ed. Madrid: Jarpyo; 2011. p. 221-8.

9. Zargar S, Kochhar R, Mehta S, Mehta SK. The role of fiberoptic endoscopy in the management of corrosive ingestion and modified endoscopic classification of burns. Gastrointest Endosc. 1991;37:165-9.

10. Donatone J. Urgencias endoscópicas digestivas en pediatría. Rev Med Clin Condes. 2009;20:869-77.

11. García A. Estenosis esofágica por ingestión de sustancias cáusticas. Rev Cub Cir. 2007:46:1-6.

12. Pedrón C, Benlloch C, Navas-López VM, Gómez-López L, Redencillas-Ferrero S, Moreno-Villares JM, et al. Documento de consenso SENPE/SEGHNP/ANECIPN/SECP sobre vías de acceso en nutrición enteral pediátrica. Nutr Hosp. 2011;26:1-15.

13. Parra-Gámez L, Reyes J, Escobar C. La desnutrición y sus consecuencias sobre el metabolismo intermedio. Rev Fac Med UNAM. 2003:46:32-6.

14. Toussaint G, García-Aranda J. Desnutrición energético-proteínica. 3. ${ }^{\mathrm{a}}$ ed. Ciudad de México: Médica Panamericana; 2015. p. 263-96.

15. Instituto Nacional de Estadística y Geografía. México: Estadísticas de mortalidad. Disponible en: http://www3.inegi.org.mx/sistemas/cni/escenario.aspx?idOrden $=1.1 \&$ ind $=6300000011 \&$ gen $=146 \& d=n$

16. Sánchez-Ramírez C, Larrosa-Haro A, Vásquez-Garibay EM, Macías-Rosales R. Socio-demographic factors associated with caustic substance ingestion in children and adolescents. Inter $\mathrm{J}$ Ped Otorhinolaryngol. 2012;76:253-6.

17. Gutiérrez JP, Rivera-Dommarco J, Shamah-Levy T, Villalpando-Hernández S, Franco A, Cuevas-Nasu L, et al. Encuesta Nacional de Salud y Nutrición 2012. Resultados nacionales. Cuernavaca, México: Instituto Nacional de Salud Pública; 2012.

18. Sánchez-Ramírez CA, Larrosa-Haro A, Vásquez-Garibay EM, Rodríguez-Anguiano AK, Cámara-López ME. Nutritional status in children with esophageal stenosis and dysphagia associated with caustic ingestion. Nutr Hosp. 2016;33:26-30. 